\title{
Understanding the fundamentals of proteomics
}

\author{
Suliman A. Alsagaby* \\ Department of Medical Laboratories Sciences, College of Applied Medical Sciences, \\ Majmaah University, PO Box 1712, Majmaah 11932, Saudi Arabia.
}

\begin{abstract}
Proteomics is an advanced approach that aims to characterize a large number of proteins (proteome) in a single assay, or single experiment. As opposed to single protein analysis using antibodybased techniques, such as western blotting, enzyme-linked immunosorbent assay (ELISA) and fluorescent microscopy, proteomics offers a valuable opportunity to study proteins "in bulk" in order to draw a clearer picture of the molecular biology of healthy and/or diseased cells. This review discusses the fundamentals of proteomics with respect to proteome complexity, reducing sample complexity by fractionation and separation methods, and protein identification based on peptide-mass and/or amino acid sequence. This review also presents a number of common proteomics workflows, the combination of some of which is likely to reveal larger proteome coverage. Both proteomics and transcriptomics enable researchers to comprehensively study the dynamic biological activities of living cells. However, each one of them has its limitations and advantages; therefore, combining the two approaches has the potential to better elucidate cellular biological processes.
\end{abstract}

KEYWORDS: proteomics, 2DE, 2DLC, ESI, MALDI, mass spectrometry.

\section{INTRODUCTION}

The idea of proteomics revolves around the premise that a large number of proteins can be

\footnotetext{
*Email id: s.alsaqaby@mu.edu.sa
}

characterized in a single assay (or single proteomics run) [1]. Proteomics is a relatively new approach that allows the investigation of different aspects of proteins, such as protein abundance, protein localization, protein interactions and post-translation modifications (PTMs), in a large scale [2]. A typical proteomics experiment passes through four major steps [3]. First is sample preparation, where proteins are extracted from tissues/cells or biological fluids. Second, the extracted proteins are resolved using gel electrophoresis. If trypsin digestion is part of the sample preparation, then the resultant peptides are separated using liquid chromatography. For proteins separated using gel electrophoresis, ingel trypsin digestion is usually employed. Third, peptide masses (precursor mass; MS) or/and precursor fragments masses (MS/MS) are measured by mass spectrometry. Fourth, MS spectra alone or with MS/MS spectra are used for protein identification by matching the observed spectra with the theoretical ones in a protein database using bioinformatics tools. Proteomics have been enabling scientists to characterize large number of proteins in a single assay, revealing novel insights into the molecular biology of normal and cancer cells $[4,5]$.

\section{Proteome complexity}

The number of protein-coding genes has been reported to reach 20,500 in human cells [6]. Alternative splicing is a process through which a single gene gives rise to multiple protein isoforms [7]. Consequently, the number of proteins expressed by human genome is believed to be greater than 
the number of protein-coding genes, indicating a complexity of human proteome [8]. Additional factors that increase the complexity of human proteome is PTMs and the wide difference in the concentration of different proteins [9]. The dynamic range has been reported to be six-fold and 10-fold for cellular proteins and plasma of humans, respectively [10]. All these factors necessitate a robust reduction of sample complexity prior to analysis by mass spectrometry in order to facilitate a larger identification coverage of proteome, especially proteins present in a sample at low abundance [11]. Various methods have been reported to be effective for reducing sample complexity. However, in general the more the sample handling the more the loss of proteins and higher the chance of manipulating the native state of proteome. Therefore, the ideal method of sample preparation is the one that lessens sample complexity to increase the chance of identifying larger number of proteins without causing protein loss or changing the native state of proteome [12].

\section{Fractionation for less complex proteome}

Reducing sample complexity can be achieved at two different stages; during sample preparation (protein extraction) and during proteins/peptide separation by electrophoresis or chromatography [13]. Sample fractionation by applying different detergents with different chemical prosperities in order to extract different protein fractions that correspond to various cellular compartments, such as cytoplasm or membranes, is a common method [14]. This strategy decreases the proteome complexity by extracting different parts of the proteome in a number of fractions, which are individually studied by mass spectrometry. The protein identifications in each fraction are then combined to represent the total proteome. This approach yields larger coverage of proteome. However, it increases the number of protein samples to be studied by mass spectrometry, as multiple protein fractions would be generated from a single sample $[14,15]$. Another method, known as sub-cellular fractionation, lessens sample complexity by targeting a specific organelle proteome, such as mitochondrial proteome, as opposed to the whole cellular proteome [16]. This approach does not only increase the probability of identifying more proteins, but also provide valuable clues about protein localization. Nevertheless, this method is laborious and yields low protein recovery; hence, either larger number of cells is required or very sensitive mass spectrometry should be used [16]. Reproducibility of the two methods, sample fractionation and subcellular fractionation, is an issue that has to be monitored, as variation from one extraction to another yields different protein identifications by mass spectrometry $[17,18]$. Protein markers are commonly used as quality control of the reproducibility. For example, tubulin is used as a marker of cytosolic fraction, whereas poly ADP ribose polymerase (PARP) is used as a marker of isolated nuclear proteome [19, 20]. These markers are usually detected using specific antibodies prior to analysis by mass spectrometry.

\section{Gel electrophoresis and liquid chromatography for less complex proteome}

The other stage where proteome complexity can be decreased is prior to the injection of sample to mass spectrometry, where proteins/peptides are resolved using electrophoresis or chromatography [13]. The separation methods rely on the different chemical characteristics of proteins/peptides [21]. For instance, proteins/peptides can be resolved according to their sizes using gel electrophoresis or size exclusion chromatography. In addition isoelectric focusing or ion exchange chromatography separates proteins/peptides based on their net charge. Another technology, namely reversed phase chromatography, separates proteins/peptides according to their solubility in water. Finally, by exploiting the binding properties of proteins/peptides, they can be resolved using affinity chromatography. All these technologies provide options of reducing sample complexity prior to analysis by mass spectrometry [22]. The selection of one or more of these technologies depends on the nature of proteomics project and on research budget.

In principle, mass spectrometry is a good technology to measure peptide mass and their fragments as long as peptides are delivered to mass analyzer in a simple state (ideally, one peptide at a time) [23]. The problem though with proteomics, is that samples are usually complex, meaning that large number of peptides reach mass spectrometry at 
one time point enabling the identification of only the most abundant peptides [23]. However, those present in low concentration are not detected. Therefore, scientists have been employing multiple separation methods in order to effectively simplify the sample for successful mass spectrometry analysis [24]. A common technique that serves this purpose is two-dimensional gel electrophoresis (2DE), where a net charge-dependent separation of proteins is conducted using isoelectric focusing technique $\left(1^{\text {st }}\right.$ dimension $)$, and then further separation by sodium dodecyl sulfate-polyacrylamide gel electrophoresis (SDS-PAGE) is made on the basis of protein masses ( $2^{\text {nd }}$ dimension) [25]. The multiple orthogonal separation of 2DE allows resolving a large number of proteins that reaches approximately 10,000 proteins [26]. This is a powerful separation capacity that has been attracting proteomics scientists, and has made 2DE a core technique for protein separation in common proteomics workflows. Nevertheless, the application of 2DE in a project that targets proteins with low concentration or poor solubility is not satisfactory [27]. Furthermore, the reproducibility of 2DE is an issue that should be optimized to obtain consistent findings [27].

Another technology that has been heavily employed as a mean of sample separation for proteomics experiments is liquid chromatography (LC) [28]. An advantage of using LC is that the targeted molecules are usually peptides rather than proteins. Therefore, in a proteomics workflow comprising LC, protein samples are subjected to tryptic digestion, and the resultant peptide mixture is resolved by an LC. This feature helps solve the challenge of separating hydrophobic proteins like membrane proteins, at least partially, as the soluble part of a protein (i.e. peptides that correspond to extracellular or intracellular domain) can be resolved by LC and successfully delivered to the mass spectrometer for identification [26]. This advantage is missed in 2DE; hence, LC may be a good choice to overcome some of the limitations of 2DE. As mentioned earlier, multiple dimensional separation is a key factor to report a large coverage of a proteome. As a result, researchers frequently utilize ion-exchange LC to resolve peptides on the basis of their net charge $\left(1^{\text {st }}\right.$ dimension $)$. Next, the resolved peptides are additionally separated according to their hydrophobicity using reversed phase LC $\left(2^{\text {nd }}\right.$ dimension). This type of peptide separation is known as 2DLC [29].

In ion exchange (IEX) LC [30], peptides are injected into an IEX column that is filled with charged matrix. The binding of peptides to the column is proportional to their $\mathrm{pH}$-dependent net charge. In other words, the more charged the peptide is the stronger the binding will be. As a result, the peptides will be differentially eluted as an increasing concentration of a mobile phase is run through the column. In this type of chromatography, the mobile phase is usually a salt solution, such as $\mathrm{NaCl}$. The peptide that has a net charge equal to zero will not bind to the column and will be eluted with "flow-through" (i.e. no need for salt ions for elution). Peptides with weak net charge have an earlier retention time (RT) compared with those possessing a strong net charge.

In reversed phase LC [31], peptides are run through a column stuffed with a matrix covered with hydrophobic alkyl chains. Depending on the solubility of peptides, they will be weakly or strongly bound to the column. Polar peptides do not bind to the column; thus, they are eluted at the "flow-through" (i.e. their retention time is zero). The column-bound peptides can be differentially eluted by passing an increasing concentration of an organic solvent such as acetonitrile or ethanol. Less polar peptides have an earlier RT compared with the non-polar peptides.

The configuration of 2DLC can be either online or off-line [32]. The online option is fully automated, where every peptide fraction eluted from IEX column by a particular salt fraction (mobile phase) is desalted in a guard column and sent directly to reversed phase column for additional separation. In contrast, the off-line mode conducts a complete separation of peptides in an IEX column using different concentration (fractions) of mobile phase (e.g. flow-through, $100 \mathrm{mM} \mathrm{NaCl}, 200 \mathrm{mM} \mathrm{NaCl}$, $400 \mathrm{mM} \mathrm{NaCl}, 600 \mathrm{mM} \mathrm{NaCl}, 800 \mathrm{mM} \mathrm{NaCl}$ and $1 \mathrm{M} \mathrm{NaCl}$ ). Peptides that are separated by each fraction of the mobile phase are collected in tubes. Next, peptides in each tube are subjected to desalting and further separation by a reversed phase LC. The off-line mode is laborious but has a greater separation capacity compared with the online 
mode [33]. The separation of peptides by 2DLC with many salt fractions (mobile phase) for the $1^{\text {st }}$ dimension (IEX column) tends to reward a bigger proteome identification [34].

\section{Protein identification by mass spectrometry}

Following peptide separation, the peptides are delivered into a mass spectrometer for mass analysis. Therefore, protein detection by mass spectrometry is a mass-based identification [35]. To determine the peptide mass, peptides must be converted into a gaseous ion; this step is known as peptide ionization. Next, ionized peptides (mostly positively charged peptides) travel through a mass analyzer, where the travel speed is dependent on the mass-to-charge $(\mathrm{m} / \mathrm{z})$ ratio of peptides. As a result, peptides with different $\mathrm{m} / \mathrm{z}$ will travel at different velocities causing them to arrive at a detector at different time points. The travel time of peptides through the mass analyzer until they hit the detector is indicative of their $\mathrm{m} / \mathrm{z}$. Peptides with large $\mathrm{m} / \mathrm{z}$ will take longer time to reach the detector compared with peptides that have small $\mathrm{m} / \mathrm{z}$ [36]. Figure 1 shows a typical MS spectra, where the $\mathrm{m} / \mathrm{z}$ ratio of a number of peptides (precursors) is determined. Although this is the basic concept of mass spectrometry, different types of the ionization source and mass analyzer are available to suit different proteomics projects [37].
There are two common methods through which peptide ionization can be achieved for proteomics [38]. First, electrospray ionization (ESI), where peptides are mixed with an organic solvent and passed through a needle-like metal tube. The sample is sprayed at the end of the needle-like tube and the solvent evaporates as a result of high electric field. This process leads to the ionization of peptides in a gaseous phase [39]. Second, matrixassisted laser desorption/ionization (MALDI), in which peptides and a matrix ( $\alpha$-cyano-4hydroxycinnamic acid; CHCA) are dissolved in an organic solvent, and deposited on a metal plate and left to dry. The matrix (CHCA) absorbs UV and as a result emits heat; therefore, when a UV beam is fired for a short time period at the sample on the metal plate the heat emitted from the matrix causes a sublimation and ionization of the peptides [40].

ESI generates multiply charged peptides, whereas the vast majority of ionized peptides by MALDI are singly charged [41]. As a result, MALDIbased ionization produces less complex spectra compared with ESI [40]. The stability of ESI flow is an issue that has to be optimized and preferably monitored because any blockage or change in the ESI flow can greatly impact on the mass spectrometry analysis [42]. In contrast, with MALDI, resolved peptides are mixed with the matrix and organic solvent and spotted on MALDI plate. Therefore,

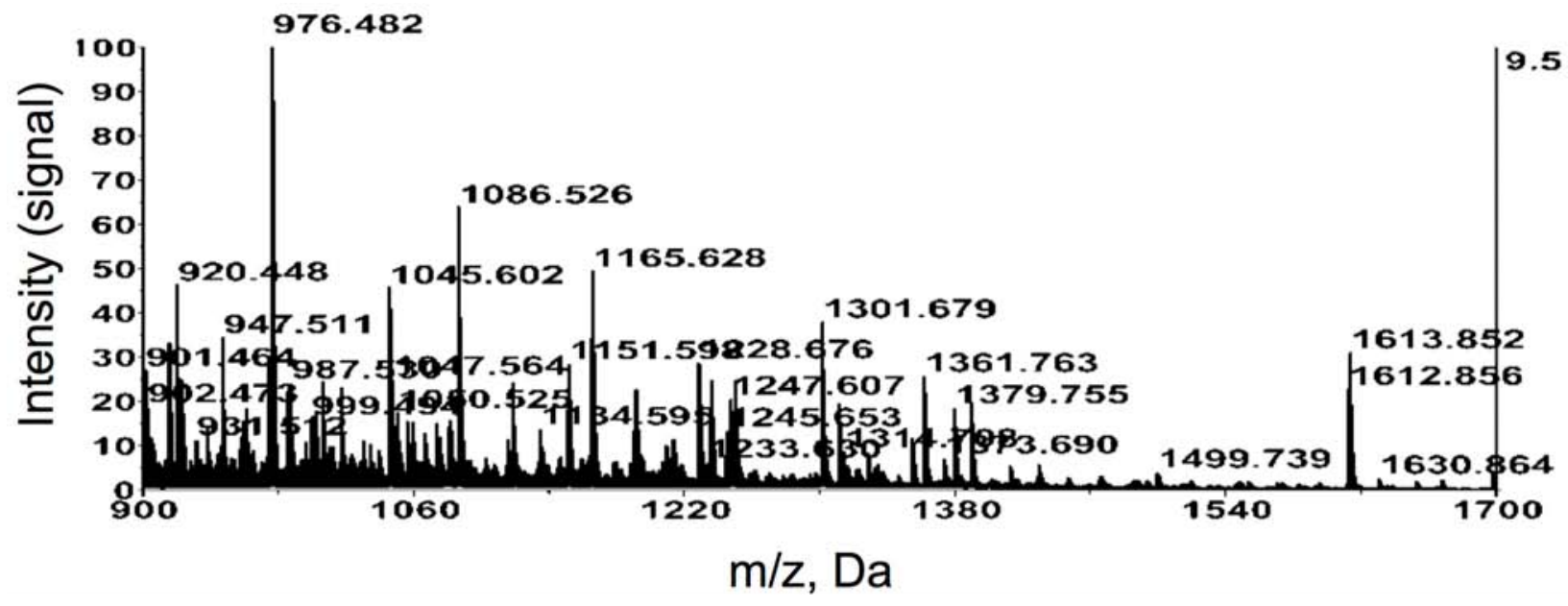

Figure 1. MS spectra of peptides that were separated by 2DLC and studied by MALDI mass spectrometry. The MS spectra show the $\mathrm{m} / \mathrm{z}$ ratio of a number of peptides that correspond to proteins extracted from human cells. 
in LC or 2DE MALDI mass spectrometry set up, there are two separate stages of analyses [18]. First, sample separation by LC or 2DE followed by preparation and deposition of the mixture of peptides with the matrix and organic solvent on MALDI plate. Second, peptide ionization and mass spectrometry analysis. In LC-ESI mass spectrometry, however, sample separation followed by ionization and mass spectrometry analysis are connected processes [43]. Therefore, all the three instruments (LC, ESI and mass spectrometry) have to be at "ready" mode prior to sample injection into LC. If a breakdown occurs to ESI or mass spectrometry, the sample cannot be recovered once injected into LC. On the other hand, with MALDI mass spectrometry, if a problem happens to the ionization step or mass spectrometry analysis, the MALDI plate can always be ejected and stored, while trouble-shooting tacks place. This is an important consideration when working with precious samples.

Although peptide mass is an important information for protein identification, it is not sufficient when the sample is a mixture of peptides that correspond to multiple proteins [44]. Therefore, for peptide mass-based protein identification, also known as peptide mass fingerprint (PMF), a protein of interest must be separated from other proteins present in the sample commonly using 2DE. Next, protein is digested by trypsin following the protein spot excision from 2DE [44]. In this situation, there is a high chance that all peptides that resulted from the tryptic digestion belong to one protein. Following the determination of the $\mathrm{m} / \mathrm{z}$ ratio of peptides using mass spectrometry, they are searched against protein database, where in silico tryptic digestion is conducted. The best match between the observed $\mathrm{m} / \mathrm{z}$ ratio (in mass spectrometry) with the theoretical $\mathrm{m} / \mathrm{z}$ ratio (in the database) enables protein identification [45].

Another type of protein identification by mass spectrometry is based on amino acid sequencing of peptides (tandem mass spectrometry; MS/MS) [18]. This kind of analysis employs additional parts of mass spectrometry known as collisioninduced cell (CID), where peptide ion (precursor) is fragmented into smaller paces (ion fragments), and a second mass analyzer, where $\mathrm{m} / \mathrm{z}$ ratio of the resultant peptide fragments are measured. This happens subsequent to the determination of the $\mathrm{m} / \mathrm{z}$ ratio of the parent peptide (Figure $2 \mathrm{~A}$ ). Three bonds of the intact peptide are targeted for breakage in a CID, resulting in six different ion fragments; a, b and $\mathrm{c}$ ions containing the c-terminal of the intact peptide, and $\mathrm{z}, \mathrm{y}$ and $\mathrm{x}$ of the ion fragments containing the $\mathrm{N}$-terminal of the intact peptide [46] (Figure 2B). However, the most common ion fragments are $\mathrm{b}$ and $\mathrm{y}$. Peptide fragmentation does not aim to "chop up" each single amino acid from the parent peptide. In contrast, it generates a number of ion fragments with different sizes (masses) and intensities as a result of insufficient peptide fragmentation. The ion fragments leave the CID travelling through a second mass analyzer to a detector. The travel time of the fragment ions is functional of their $\mathrm{m} / \mathrm{z}$ ratio [37]. The output of this analysis is MS/MS spectra, through which amino acid sequencing of a peptide can be made. Typical MS/MS spectra show various fragments of the parent peptide with different signals and $\mathrm{m} / \mathrm{z}$ ratio (Figure $2 \mathrm{C}$ ). The difference between two adjacent ion fragments (y or $\mathrm{b}$ ions) gives the mono-isotopic mass of an amino acid [46]. The observed MS/MS spectra are matched with theoretical MS/MS spectra in a protein database, and the best match provides the amino sequencing of the parent peptide [47], which in turn gives evidence of a protein identification (Figure 2D).

In contrast to amino acid sequencing (MS/MS) method, PMF-based protein identification is faster, less expensive, generates less data (only MS spectra), and does not require CID or a second mass analyzer [45]. However, amino acid sequencing approach provides other information of the peptide in addition to its $\mathrm{m} / \mathrm{z}$ (both MS and MS/MS), yielding more evidences for peptide identification [47]. For example, different peptides from different proteins may have the same $\mathrm{m} / \mathrm{z}$ ratio, causing an ambiguity for protein identification using the PMF method. On the other hand, this ambiguity can be removed as additional information of the peptides, which is amino acid sequence (MS/MS), is provided. Another drawback of the PMF method is its limitation to the identification of peptides that correspond to a single protein; hence, protein purification has to be conducted prior to PMF [45]. In contrast, protein identification by 
(A)
Determination of peptides
$\mathrm{m} / \mathrm{z}$ ratio in the $1^{\text {st }}$ mass
analyzer (MS spectra)
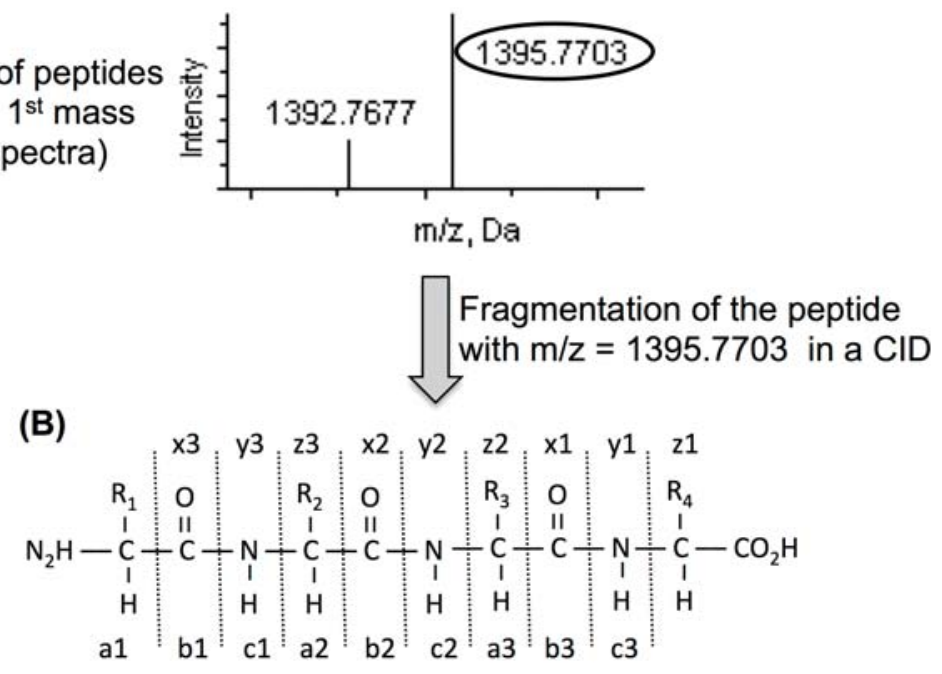

Determination of the $\mathrm{m} / \mathrm{z}$ ratio of the peptide fragments in a second mass analyzer.

(C)

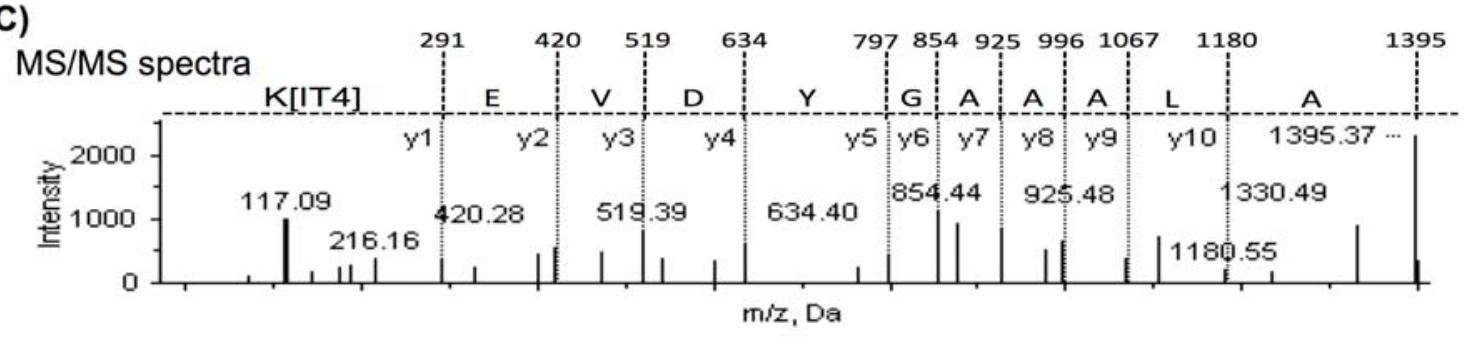

(D)

Histone H1.2 (UniProt identifier P16403)
MSETAPAAPAAAPPAEKAPVKKKAAKKAGGTPRKASGPPVSELITKAVAASKERSGVSLAALKKALAAAGYDVEKN
NSRIKLGLKSLVSKGTLVQTKGTGASGSFKLNKKAASGEAKPKVKKAGGTKPKKPVGAAKKPKKAAGGATPKKSAKKTP
KKAKKPAAATVTKKVAKSPKKAKVAKPKKAAKSAAKAVKPKAAKPKVVKPKKAAPKKK

Figure 2. Amino acid sequencing-based protein identification (tandem mass spectrometry; MS/MS spectra). Digested peptides are separated by 2DLC and subjected to MALDI mass spectrometry analysis. The $\mathrm{m} / \mathrm{z}$ ratio of peptides (MS spectra) is determined in the MS mode (A). Fragmentation of the peptide with m/z ratio 1395.7703 is conducted using a CID (B). The $\mathrm{m} / \mathrm{z}$ ratio of the peptide fragments (MS/MS spectra) is determined (C). Amino acid sequence-based protein identification is performed by matching the observed spectra with theoretical spectra stored in a proteins database using bioinformatics tools; the sequenced peptide (ALAAAGYDVEK) belongs specifically to histone H1.2 (D).

tandem mass spectrometry (MS/MS) can be conducted for a mixture of peptides of different proteins. Given the advantage of tandem mass spectrometry (MS/MS) over the PMF (MS) with respect to protein identification, the former is widely used compared with the latter [37].

\section{Method options for proteomics}

Various experimental options of proteomics are available. For example, 2DE-MS, where proteins are resolved in a 2DE followed by tryptic digestion and PMF-based protein identification [48]. Another approach is 2DE-MS/MS, which is 
similar to the 2DE-MS, but the protein identification is based on amino acid sequence of peptides (tandem mass spectrometry) rather than by PMF [49]. GeLC-MS or GeLC-MS/MS employs SDS-PAGE followed by LC for protein/peptide separation and PMF or tandem mass spectrometry for protein(s) identification [50]. In gel-free proteomics workflows, such as 1DLC-MS/MS or 2DLC-MS/MS, peptides are resolved by 1DLC or 2DLC and identified by tandem mass spectrometry [51, 52]. These workflows are complementary to each other; thus, using more than one workflow may increase the proteome coverage.

\section{Combining proteomics with transcriptomics}

The notion of the global profiling of biological molecules that indicate the dynamic activity of living cells was made possible by the transcriptomics approach, where the whole transcriptome can be relatively measured in a single assay [53]. Later, proteomics was introduced as a complementary approach and to overcome some limitations of transcriptomics [43]. As proteins are the main functional molecules in living cells, proteomics provides a direct mean to measure protein abundance rather than inferring that from mRNA expression using transcriptomics [22]. PTMs, such as protein phosphorylation, are key determinants of protein activity. Unlike transcriptomics, proteomics allows the identification of PTMs [54]. Organelle proteomics is a very powerful application that yields accurate data about protein localization, which is not possible to obtain using transcriptomics [55]. Nevertheless, given the amplification step within a protocol of a typical transcriptomics experiment and the DNA-cRNA or DNA-cDNA hybridization-based measurement of transcriptome, transcriptomics is still ahead of proteomics with respect to coverage [56]. In principle, transcriptomics allows the determination of all expressed genes in living cells [56]. In contrast, low abundant proteins and those with poor solubility are challenging to identify/quantify using proteomics limiting the identification coverage of proteomics [57]. As a result, combining the two approaches provides deeper and wider information about the molecular biology of living cells [58-60].

\section{CONFLICT OF INTEREST STATEMENT}

The author declares no conflict of interest.

\section{ABBREVIATIONS \\ 2DE : Two-dimensional gel electrophoresis \\ 2DLC : Two-dimensional liquid chromatography \\ ESI : Electrospary ionization \\ MALDI : Matrix-assisted laser desorption/ionization \\ MS : Mass spectrometry \\ MS/MS : Tandem mass spectrometry \\ CID : Collision induced cell}

\section{REFERENCES}

1. Collins, B. C. and Aebersold, R. 2018, Nat. Biotechnol., 36, 1051-1053.

2. Anderson, N. L., Matheson, A. D. and Steiner, S. 2000, Curr. Opin. Biotechnol., 11, 408-412.

3. Tyers, M. and Mann, M. 2003, Nature, 422, 193.

4. Haudek, V. J., Slany, A., Gundacker, N. C., Wimmer, H., Drach, J. and Gerner, C. 2009, J. Proteome Res., 8, 3834-3843.

5. Alsagaby, S. A. and Alhumaydhi, F. A. 2019, Saudi Med. J., 40, 317-327.

6. Clamp, M., Fry, B., Kamal, M., Xie, X., Cuff, J., Lin, M. F., Kellis, M., LindbladToh, K. and Lander, E. S. 2007, Proceedings of the National Academy of Sciences, 104, 19428-19433.

7. Modrek, B. and Lee, C. 2002, Nat. Genet., 30, 13.

8. Blencowe, B. J. 2017, Trends Biochem. Sci., 42, 407-408.

9. Aebersold, R., Agar, J. N., Amster, I. J., Baker, M. S., Bertozzi, C. R., Boja, E. S., Costello, C. E., Cravatt, B. F., Fenselau, C. and Garcia, B. A. 2018, Nat. Chem. Biol., 14, 206.

10. Ponomarenko, E. A., Poverennaya, E. V., Ilgisonis, E. V., Pyatnitskiy, M. A., Kopylov, A. T., Zgoda, V. G., Lisitsa, A. V. and Archakov, A. I. 2016, Int. J. Anal. Chem., 2016, 7436849.

11. Chandramouli, K. and Qian, P.-Y. 2009, Human Genomics and Proteomics: HGP, 2009, 239204.

12. Feist, P. and Hummon, A. 2015, International Journal of Molecular Sciences, 16, 3537-63. 
13. Canas, B., Pineiro, C., Calvo, E., LópezFerrer, D. and Gallardo, J. M. 2007, J. Chromatogr. A, 1153, 235-258.

14. Alsagaby, S. A., Khanna, S., Hart, K. W., Pratt, G., Fegan, C., Pepper, C., Brewis, I. A. and Brennan, P. 2014, J. Proteome Res., 13, 5051-5062.

15. Ahmed, F. E. 2009, J. Sep. Sci., 32, 771798.

16. Gatto, L., Vizcaíno, J. A., Hermjakob, H., Huber, W. and Lilley, K. S. 2010, Proteomics, 10, 3957-3969.

17. Yan, W., Aebersold, R. and Raines, E. W. 2009, J. Proteomics, 72, 4-11.

18. Yates, J. R., Ruse, C. I. and Nakorchevsky, A. 2009, Annu. Rev. Biomed. Eng., 11, 4979.

19. Alsagaby, S., Brewis, I., Pepper, C., Fegan, C. and Brennan, P. 2010, Immunology, 131, 115.

20. Brennan, P., Shore, A. M., Clement, M., Hewamana, S., Jones, C. M., Giles, P., Fegan, C., Pepper, C. and Brewis, I. A. 2009, PROTEOMICS-Clinical Applications, 3, 359-369.

21. Aslam, B., Basit, M., Nisar, M. A., Khurshid, M. and Rasool, M. H. 2017, J. Chromatogr. Sci., 55, 182-196.

22. Larance, M. and Lamond, A. I. 2015, Nature Reviews Molecular Cell Biology, 16, 269.

23. Bantscheff, M., Schirle, M., Sweetman, G., Rick, J. and Kuster, B. 2007, Anal. Bioanal. Chem., 389, 1017-1031.

24. Zhang, X., Fang, A., Riley, C. P., Wang, M., Regnier, F. E. and Buck, C. 2010, Anal. Chim. Acta, 664, 101-113.

25. Rabilloud, T., Chevallet, M., Luche, S. and Lelong, C. 2010, J. Proteomics, 73, 20642077.

26. Issaq, H. J. 2001, Electrophoresis, 22, 36293638.

27. Oliveira, B. M., Coorssen, J. R. and Martinsde-Souza, D. 2014, J. Proteomics, 104, 140150.

28. Motoyama, A. and Yates Iii, J. R. 2008, Analytical Chemistry, 80, 7187-7193.

29. Hinzke, T., Kouris, A., Hughes, R.-A., Strous, M. and Kleiner, M. 2019, Frontiers in Microbiology, 10, 238.
30. Manadas, B., Mendes, V. M., English, J. and Dunn, M. J. 2010, Expert Review of Proteomics, 7, 655-663.

31. Xie, F., Smith, R. D. and Shen, Y. 2012, J. Chromatogr., 1261, 78-90.

32. Wu, Q., Yuan, H., Zhang, L. and Zhang, Y. 2012, Anal. Chim. Acta, 731, 1-10.

33. Bereszczak, J. Z. and Brancia, F. L. 2009, Comb Chem High Throughput Screen, 12, 185-193.

34. Havugimana, P. C., Wong, P. and Emili, A. 2007, J. Chromatogr. B, 847, 54-61.

35. Aebersold, R. and Mann, M. 2003, Nature, 422, 198.

36. Mann, M. 2016, Nature Reviews Molecular Cell Biology, 17, 678.

37. Han, X., Aslanian, A. and Yates Iii, J. R. 2008, Curr. Opin. Chem. Biol., 12, 483-490.

38. Cañas, B., López-Ferrer, D., Ramos-Fernández, A., Camafeita, E. and Calvo, E. 2006, Briefings in Functional Genomics, 4, 295-320.

39. Awad, H., Khamis, M. M. and El-Aneed, A. 2015, Applied Spectroscopy Reviews, 50, 158-175.

40. El-Aneed, A., Cohen, A. and Banoub, J. 2009, Applied Spectroscopy Reviews, 44, 210-230.

41. Domon, B. and Aebersold, R. 2006, Science, 312, 212-217.

42. Wilm, M. 2011, Mol. Cell Proteomics, 10, M111.009407.

43. Walther, T. C. and Mann, M. 2010, The Journal of Cell Biology, 190, 491-500.

44. Thiede, B., Höhenwarter, W., Krah, A., Mattow, J., Schmid, M., Schmidt, F. and Jungblut, P. R. 2005, Methods, 35, 237-247.

45. Henzel, W. J., Watanabe, C. and Stults, J. T. 2003, J. Am. Soc. Mass Spectrom., 14, 931942.

46. Lim, M. S. and Elenitoba-Johnson, K. S. J. 2004, Lab. Invest., 84, 1227.

47. Steen, H. and Mann, M. 2004, Nature Reviews Molecular Cell Biology, 5, 699.

48. Voss, T., Ahorn, H., Haberl, P., Döhner, H. and Wilgenbus, K. 2001, Int. J. Cancer, 91, 180-186.

49. Perrot, A., Pionneau, C., Nadaud, S., Davi, F., Leblond, V., Jacob, F., Merle-Béral, H., Herbrecht, R., Béné, M.-C. and Gribben, J. G. 2011, Blood, 118, e1-e15. 
50. Miguet, L., Béchade, G., Fornecker, L., Zink, E., Felden, C., Gervais, C., Herbrecht, R., van Dorsselaer, A., Mauvieux, L. and Sanglier-Cianferani, S. 2009, J. Proteome Res., 8, 3346-3354.

51. Barnidge, D. R., Jelinek, D. F., Muddiman, D. C. and Kay, N. E. 2005, J. Proteome Res., 4, 1310-1317.

52. Eagle, G. L., Zhuang, J., Jenkins, R. E., Till, K. J., Jithesh, P. V., Lin, K., Johnson, G. G., Oates, M., Park, K. and Kitteringham, N. R. 2015, Mol. Cell Proteomics, 14, 933-945.

53. Cox, J. and Mann, M. 2007, Cell, 130, 395398.

54. Aebersold, R. and Mann, M. 2016, Nature, 537, 347.
55. Thul, P. J., Åkesson, L., Wiking, M., Mahdessian, D., Geladaki, A., Blal, H. A., Alm, T., Asplund, A., Björk, L. and Breckels, L. M. 2017, Science, 356, eaal3321.

56. Lowe, R., Shirley, N., Bleackley, M., Dolan, S. and Shafee, T. 2017, PLoS Comput. Biol., 13, e1005457.

57. Schubert, O. T., Röst, H. L., Collins, B. C., Rosenberger, G. and Aebersold, R. 2017, Nat. Protoc., 12, 1289.

58. Buescher, J. M. and Driggers, E. M. 2016, Cancer \& Metabolism, 4, 4.

59. Alsagaby, S. A. 2019, Majmaah Journal of Health Sciences, 7, 1-22.

60. Alsagaby, S. A. 2019, Saudi Med. J., 40, 328-338. 